\title{
FREE TOPOLOGICAL GROUPS
}

\author{
CARLOS R. BORGES
}

(Received 19 January 1976; revised 10 August 1976)

\begin{abstract}
Let $X$ be any Tychonoff space and $\beta X$ the Stone-Čech compactification of $X$. Let $F(\beta X)$ be the Graev free group of $\beta X$ and let $\mathcal{G}^{\prime}$ be the subspace topology on the Graev group $F(X)$. Our results demonstrate that this topology is useful and behaves extremely well; the behavior of the free topology still remains enigmatic.

There are various applications, some of which clarify the free topology on $F(X)$, while others improve various results recently published.
\end{abstract}

Let $X$ be any Tychonoff space and $\beta X$ the Stone-Čech compactification of $X$. Let $F(\beta X)$ be the Graev free group of $\beta X$ and let $\left(\mathcal{S}^{\prime}\right.$ be the subgroup topology on the Graev group $F(X)$. Our results demonstrate that this topology is useful and behaves extremely well (see Lemma 2.2 and Theorem 2.3); the behavior of the free topology still remains enigmatic.

There are various applications, some of which clarify the free topology on $F(X)$ (see Theorem 2.4), while others improve various results recently published by B. V. S. Thomas (see Propositions 3.1, 3.2 and 3.3).

\section{Terminology and preliminaries}

For the sake of completeness, we will recall some of Graev's terminology .nd introduce some of our own. We deal only with Graev's non-abelian free :opological groups, inasmuch that it is easily seen that our methods also apply ro the abelian case and to Markov's free topological groups, and to discuss these minor details would only detract from the task at hand.

Definition 1.1. Let $X$ be a Tychonoff space. We distinguish in $X$ an arbitrary point $\theta$. A free topological group of the space $X$ is a topological group $H$ with the following properties:

(1) $X$ is a closed subspace of $H$,

(2) $X$ generates $H$ topologically (i.e., no proper closed subgroup of $H$ contains $X)$, 
(3) If $\psi$ is any continuous function from $X$ to any topological group $G$, which carries $\theta$ to the unit element of $G$, then there exists a continuous homomorphism $\Phi: H \rightarrow G$ such that $\Phi \mid X=\psi$.

By Theorems 1 and 2 of Graev (1962), for each Tychonoff space $(X, \tau)$ there exists a unique (up to isomorphism) free Graev topological group, which we will denote by $\left(F(X),(\mathfrak{S}) . F(X)\right.$ denotes the free algebraic group $F_{Y}$ (of reduced words) generated by $Y=X-\{\theta\}$, with $\theta$ and the unit element of $F_{Y}$ identified; ( $(\mathfrak{S}$ denotes the Graev free group topology on $F(X)$.

Throughout, we will adhere to the following terminology:

(a) Let $F_{1}(X)=X \cup X^{-1}$, where $X^{-1}$ is the set of all symbols $x^{-1}$ with $x \in Y$; for $n \geqq 2$, let $F_{n}(X)$ be the set of those elements of the group $F(X)$ which have reduced length (with respect to the basis $Y$ ) not greater than $n$. Whenever feasible, we let $F_{n}(X)=F_{n}$ for $n=1,2, \cdots$.

(b) For any space $X$ and positive integer $n$, let $X^{n}$ be the cartesian product of $n$ copies of $X$ with the product topology.

(c) For any family $\left\{X_{\alpha}\right\}_{\alpha \in A}$ of spaces, let $V_{\alpha} X_{\alpha}$ be the disjoint topological union of the spaces $X_{\alpha}$ with $\alpha \in \Lambda$.

(d) Given a sequence $\left\{\left(X_{n}, \tau_{n}\right)\right\}_{n=1}^{\infty}$ of spaces such that each $X_{n}$ is a closed subspace of $X_{n+1}$, let $\Sigma_{n} X_{n}$ be $X=\bigcup_{n=1}^{\infty} X_{n}$ with the weak topology. (i.e., $A \subset X$ is open if and only if $A \cap X_{n} \in \tau_{n}$, for each $n$.) Throughout, the equality $X=\Sigma_{n} X_{n}$ will mean that each $X_{n}$ is a subspace of $X$ and that $X$ has the topology of $\Sigma_{n} X_{n}$.

(e) For any space $X$, we let $p_{X}: V_{n}\left(F_{1}(X)\right)^{n} \rightarrow F(X)$ and $q_{X}: V_{n} F_{n}(X) \rightarrow F(X)$ be the natural maps

$$
\text { (i.e., } \left.p_{X}\left(x_{1}, \cdots, x_{m}\right)=x_{1} x_{2} \cdots x_{m}, q_{x}\left(a_{1} a_{2} \cdots a_{m}\right)=a_{1} a_{2} \cdots a_{m}\right) \text {. }
$$

Whenever feasible, we let $p_{x}=p$ and $q_{x}=q$. Throughout, whenever we refer to the maps $p$ and $q$ we automatically assume that each $F_{n}(X)$ inherits the topology of $F(X)$. For $k=1,2, \cdots$, we let $p_{k}=p \mid\left(F_{1}(X)\right)^{k}$ and $K_{k}(X)=$ $p_{k}^{-1}\left(F_{k}-F_{k-1}\right)$. We also let $\tau_{p}$ and $\tau_{q}$ denote the quotient topologies on $F(X)$ with respect to the maps $p$ and $q$, respectively.

According to Michael (1968), a space $X$ is called a $k_{\omega}$-space provided that $X=\Sigma_{n} X_{n}$, with each $X_{n}$ compact. Part (a) of the following result is essentially proved by Mack, Morris and Ordman (1973). (To be precise, they proved that $\tau_{q}=(\mathbb{S}$; however, it is easily seen that similar techniques will prove that $\left(\mathfrak{S}=\tau_{p}\right.$.) Part $(\mathrm{b})$ is obvious, but very useful.

Proposition 1.2. (a) for any Hausdorff $k_{\omega}$-space $(X, \tau), B=\tau_{p}=\tau_{q}$.

(b) If $(X, \tau)$ is compact Hausdorff, the maps $p_{k}$ are closed continuous functions. 


\section{Other topologies on free groups}

Throughout this section, let $X$ be any Tychonoff space and $\beta X$ the Stone-Cech compactification of $X$. Give $F(X)$ the subspace topology (S)' it inherits from $(F(\beta X),(S)$. The following results exhibit the simplicity and usefulness of this topology.

LEMMA 2.1. The natural map $q: V_{n} F_{n}(\beta X) \rightarrow(F(\beta X)$, (G) $)$ is a hereditarily quotient map.

Proof. By Lemma 5.2(c) of Michael (1972), it suffices to prove that, for each $A \subset F(\beta X)$,

$$
y \in A^{-} \text {implies } q^{-1}(y) \cap q^{-1}(A)^{-} \neq \varnothing:
$$

Say not. Say $z \in A^{-}$with $q^{-1}(z) \cap q^{-1}(A)^{-}=\varnothing$. Also, say $z \in F_{m}-F_{m-1}$. Keeping in mind that each $F_{n}$ is both a subspace of $F(\beta X)$ and of $V_{n} F_{n}(\beta X)$, let us now construct sets $N_{m}, N_{m+1}, \cdots$ such that

(i) each $N_{m+j}$ is an open subset of $F_{m+j} \subset F(\beta X) \cap V_{n} F_{n}(\beta X)$,

(ii) each $N_{m+j+1} \cap F_{m+j}=N_{m+j}$,

(iii) $z \in N_{m+j}$ and $N_{m+j}^{-} \cap q^{-1}(A)^{-}=\varnothing$, for $j=0,1, \cdots$.

Let $N_{m}$ be some open subset of $F_{m}-F_{m-1}$ with $z \in N_{m}$ and $N_{m}^{-1} \cap q^{-1}(A)^{-}=$ $\varnothing$ (note that $F_{m}$ is normal, by Proposition 1.2(b); also $z \notin F_{m-1}$ and $F_{m-1}$ is a closed subspace of $F_{m}$ ). Clearly $N_{m}$ satisfies (i) and (iii).

Assuming we already have sets $N_{m}, \cdots, N_{m+k}$ which satisfy (i), (ii) and (iii), let us construct a set $N_{m+k+1}$ which satisfies (i), (ii) and (iii). Let $U$ be an open subset of $F_{m+k+1}$ such that $U \cap F_{m+k}=N_{m+k}$. Since $F_{m+k+1}$ is normal, also let $W$ be an open subset of $F_{m+k+1}$ such that

$$
N_{m+k}^{-} \subset W, W^{-} \cap q^{-1}(A)^{-}=\varnothing .
$$

It is immediately seen that the set $N_{m+k+1}=U \cap W$ satisfies conditions (i), (ii) and (iii). This completes the induction.

Finally, letting

$$
N=\bigcup_{j=0}^{\infty} N_{m+i}
$$

it is easily seen that $N=q^{-1} q(N)$ is open and $q^{-1}(z) \subset N$ (note that $q^{-1}(z) \cap F_{m+j}=\{z\}$, for $\left.j=0,1, \cdots\right)$ and $N \cap q^{-1}(A)=\varnothing$. It follows that $q(N)$ is an open neighborhood of $z$ with $q(N) \cap A=\varnothing$, a contradiction. This completes the proof.

LEMMA 2.2. $\left(F(X),{\left(S^{\prime}\right)}^{\prime}\right.$ is a topological group such that

(a) $X$ and each $F_{n}(X)$ are closed subspaces of $\left(F(X), \uplus^{\prime}\right)$. 
(b) Each $p_{n} \mid K_{n}$ is a homeomorphism.

(c) The natural map $q: V_{n} F_{n}(X) \rightarrow\left(F(X),\left(\mathcal{G}^{\prime}\right)\right.$ is compact-covering.

Proof. To prove (a) simply observe that $X=\beta X \cap F(X)$ and $F_{n}(X)=$ $F_{n}(\beta X) \cap F(X)$.

Part (b). From Proposition $1.2(\mathrm{~b})$, it is immediate that $p_{n} \mid K_{n}(\beta X)$ is a one-to-one quotient map; therefore it is a homeomorphism. It follows that $p_{n} \mid K_{n}(X)$ is also a homeomorphism.

Part (c). Obviously, $q$ is continuous. Let $K$ be a compact subspace of $F(X)$. Then $K$ is a compact subspace of $F(\beta X)$ and, by Proposition 2.7 of Hyman (1968), $K \subset$ some $F_{n}(\beta X)$. Therefore $K \subset$ some $F_{n}(X)=$ $F_{n}(\beta X) \cap F(X)$, which implies that $K$ is a compact subset of $V_{n} F_{n}(X)$, and therefore that $q$ is compact-covering.

TheOREM 2.3. $\left(F(X),\left(S^{\prime}\right)\right.$ is a topological group, such that

(a) $\left(F(X), \mathbb{S}^{\prime}\right)=\Sigma_{n} F_{n}(X)$, with each $F_{n}(X)$ a subspace of $\left(F(X),\left(\mathcal{S}^{\prime}\right)\right.$.

(b) If $\psi$ is any continuous function from $X$ to any compact topological group $G$, which carries $\theta$ to the unit element of $G$, then there exists a continuous homomorphism $\Phi:\left(F(X),\left(\mathcal{S}^{\prime}\right) \rightarrow G\right.$ such that $\Phi \mid X=\psi$.

Proof. Part (a). From Lemma 2.1, the natural map $q_{X}: V_{n} F_{n}(X) \rightarrow\left(F(X),\left(\mathcal{S}^{\prime}\right)\right.$ is a quotient map, since $V_{n} F_{n}(X)=q_{\beta X}^{-1}(F(X))$ and $q_{X}=q_{\beta X} \mid V_{n} F_{n}(X)$. From Proposition 2.5 of Hyman (1968) we then get that $\left(F(X),\left(\mathcal{S}^{\prime}\right)=\Sigma_{n} F_{n}(X)\right.$.

Part (b). This is a straightforward application of the Stone-Čech compactification.

Following the techniques of Markov (1962), Lemma 2.2 yields some new and useful facts about free topological groups of Tychonoff spaces.

THEOREM 2.4. ( $F(X),(B)$ is a topological group such that .

(a) the natural map $q: V_{n} F_{n}(X) \rightarrow(F(X)$, (B) is compact covering.

(b) If $\left(F(X),(G)\right.$ is a $k$-space then $\left(\mathbb{S}=\tau_{q}\right.$.

(c) Each $p_{n} \mid K_{n}$ is a homeomorphism. (Stated, without proof, in Arhangel'skii (1968).)

Proof. Recall that (S) is the supremum of all group topologies $\tau$ for $F(X)$ such that $X$ is a subspace of $(F(X), \tau)$. Part (a) is now a straightforward consequence of Lemma 2.2 (c), inasmuch that $q$ is obviously continuous and $\left(F(X),(S)\right.$ has no more compact subspaces than $\left(F(X),\left(\mathcal{S}^{\prime}\right)\right.$.

To prove (b), note that from Lemma 11.2 of Michael (1966) and part (a), it follows that the map $q$ is a quotient map; that is, $\mathbb{F}=\tau_{q}$.

Part (c). Let $\tau_{1}$ (resp. $\tau_{2}$ ) be the subspace topology on $F_{n}-F_{n-1}$ with respect to (G) (resp. (G') on $F(X)$. Clearly $p_{n} \mid K_{n}: K_{n} \rightarrow\left(F_{n}-F_{n-1}, \tau_{1}\right)$ is 
continuous (see Lemma 7.1 of Graev (1962)). But $\left(p_{n} \mid K_{n}\right)^{-1}$ is also continuous because of Lemma 2.2 (b) and $\tau_{2} \subset \tau_{1}$. This does the trick.

\section{Applications}

The first application extends Proposition 3.9 of Thomas (1974) to all free groups. The second extends his main theorem to the non-abelian free groups, with a much simpler proof which does not apply to the abelian case (an elementary proof of both the abelian and non-abelian cases appears in Hardy, Morris, Thompson (to appear)). The third is Joiner's Fundamental Lemma (see also the previous paper). The last discusses some separation properties of the topologies $(S)$ and $\mathbb{S}^{\prime}$.

Proposition 3.1. Let $f: X \rightarrow Y$ be a quotient onto map. Then the natural extension $\bar{f}: F(X) \rightarrow F(Y)$ is also a quotient map, whenever both groups have either the toplogy (S) or (B'. (This remains valid for the abelian case, whenever both groups have the topology (B).)

Proof. (We will consider only the case (S). The case (S') is similar because of the functorial properties of $\beta X$ ). Let $\tau_{\bar{f}}$ be the quotient topology on $F(Y)$ with respect to $\bar{f}$. Clearly $(B) \tau_{f}$. From the following diagram

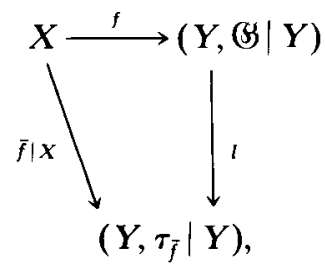

where $l$ is the identity function, one easily sees that $l$ is a homeomorphism $\left(l^{-1}\right.$ is clearly continuous since $\tau_{\bar{f}} \mid Y \supset$ (S $\mid Y ; l$ is continuous because $f$ is quotient and $\bar{f} \mid X$ is continuous). Therefore, the identity homomorphism

$$
\bar{l}:\left(F(Y),(\mathbb{G}) \rightarrow\left(F(Y), \tau_{\bar{f}}\right)\right.
$$

is continuous; that is, $\tau_{\bar{f}} \subset \mathcal{G}$, which completes the proof. (For the abelian case recall that, by 4.B of Graev (1962), the abelian free group $A(X)$ is a quotient image of $F(X)$, with respect to (S.)

Proposition 3.2. For each $n=1,2, \cdots, X^{n}$ is a subspace of $F_{n}(X)$, with respect to (3) or 'S'.

Proof. Clearly $X^{n} \subset K_{n}(X)$, for each $n$. Because of Lemma 2.2 (b) and Theorem 2.4 (c), we are done. 
Proposition 3.3 (Joiner's Fundamental Lemma). Let $X$ be any Tychonoff space and $x_{1}{ }_{1} \cdots x$ be any reduced word of $\left(F(X),(B)\right.$ or $\left(F(X),\left(S^{\prime}\right)\right.$ with $x_{i} \in X$ and $\varepsilon_{i}= \pm 1$. Then the family of all subsets of $F_{n}(X)$ of the form $U_{1}^{\varepsilon_{1}} \cdots U_{n}^{\varepsilon_{n}}$, where each $U_{i}$ is a neighborhood of $x_{i}$ in $X$, is a neighborhood base for $x_{1}^{e_{1}} \cdots x_{n}^{\varepsilon_{n}}$ in the subspace $F_{n}(X)$ of either group. (This remains valid for the abelian Graev group $(A(X),(5)$.)

Proof. Immediate from Lemma 2.2 (b) and Theorem 2.4 (c). (The abelian case follows from 4.B of Graev (1962).)

Proposition 3.4. The following implications are valid.

(a) If $X$ is a $k_{u \times m}$-space (i.e., $X=\Sigma_{n} X_{n}$ with each $X_{n}$ compact metrizable), then $\left(F(X),(G)\right.$ is a $k_{\omega c m}$-space (hence, $(F(X),(B)$ is stratifiable). (False for (B', because of the last $\S$ of Hardy, Morris and Thompson (to appear).)

(b) If $X$ is a Tychonoff cosmic (i.e., the continuous image of a separable metrizable space $)$ space then $\left(F(X),\left(\mathcal{S}^{\prime}\right)\right.$ and $(F(X),(\mathfrak{S})$ are cosmic spaces.

Proof. Part (a). Immediate from the proof of Theorem 1 of Mack, Morris and Ordman (1973), because the continuous image of a compact metrizable space is compact metrizable. The stratifiability follows from Borges (1966).

Part (b). Straightforward, since the natural map $p: V_{n}\left(F_{1}\right)^{n} \rightarrow F(X)$ is continuous.

\section{References}

A. V. Arhangel'skii (1968), 'Mappings related to topological groups', Soviet Math. Dokl. 9, 1011-1015.

C. R. Borges (1966), 'On stratifiable spaces', Pacific J. Math. 17, 1-16.

M. I. Graev (1962), 'Free topological groups', Amer. Math. Soc. Transl. (ser. 1), 8, $305-364$.

J. P. Hardy, S. A. Morris and H. B. Thompson (to appear), 'Applications of the Stone-Čech compactification to free topological groups'.

D. M. Hyman (1968), 'A category slightly larger than the metric and $C W$-categories', Michigan Math. J. 15, 193-214.

J. Mack, S. A. Morris and E. T. Ordman (1973), 'Free topological groups and the projective dimension of a locally compact abelian group', Proc. Amer. Math. Soc. 40, 303-308.

A. A. Markov (1962), 'On free topological groups', Amer. Math. Soc. Transl. (ser. 1) 8, $195-272$.

E. A. Michael (1968), 'Biquotient maps and cartesian products of quotient maps', Ann. Inst. Fourier, Grenoble, 18, 2, 287-302.

E. A. Michael (1966), ' $\aleph_{0}$-spaces', J. Math. Mech. 15, 983-1002.

E. A. Michael (1972), 'A quintuple quotient quest', Gen. Top. \& Appl. 2, 91-138.

B. V. S. Thomas (1974), 'Free topological groups', Gen. Top. \& Appl. 4, 51-72.

University of California, Davis,

California 95616,

U.S.A. 